\title{
Polymer co-crystalline films for photonics
}

\section{Christophe Daniel}

\author{
Alexandra R. Albunia \\ Concetta D'Aniello
}

Paola Rizzo

Vincenzo Venditto

Gaetano Guerra

gguerra@unisa.it

\begin{abstract}
Dipartimento di Chimica and INSTM Research Unit, University of Salerno, Via Ponte Don Melillo, 84084 Fisciano (SA), Italy

Dipartimento di Chimica and INSTM Research Unit, University of Salerno, Via Ponte Don Melillo, 84084 Fisciano (SA), Italy

Dipartimento di Chimica and INSTM Research Unit, University of Salerno, Via Ponte Don Melillo, 84084 Fisciano (SA), Italy

Dipartimento di Chimica and INSTM Research Unit, University of Salerno, Via Ponte Don Melillo, 84084 Fisciano (SA), Italy

Dipartimento di Chimica and INSTM Research Unit, University of Salerno, Via Ponte Don Melillo, 84084 Fisciano (SA), Italy

Dipartimento di Chimica and INSTM Research Unit, University of Salerno, Via Ponte Don Melillo, 84084 Fisciano (SA), Italy
\end{abstract}

Robust syndiotactic polystyrene (SPS) films exhibiting highly stable co-crystalline phases can be obtained with a large variety of lowmolecular-mass chromophore guest molecules. In this report different aspects relative to the structure and the optical properties of these films are described. In particular, possible applications of these films as fluorescent materials, as optical memories (based on the cocrystallization of photoreactive guest molecules), as non-linear optical materials (with polar guests) and as chiro-optical memories (based on temporary co-crystals with chiral guest molecules) are presented. [DOI: 10.2971/je0s.2009.09037]

Keywords: polymer co-crystals, syndiotactic polystyrene, chromophore three-dimensional orientation

\section{INTRODUCTION}

In several cases, polymeric materials with optoelectronic and photonic properties are usually simply based on the dispersion of chromophores in an amorphous polymer matrix although in order to reduce the diffusivity, the chemical bonding to the polymer backbone (grafting), or the inclusion of the chromophores in the polymer chain as monomers (polymerization) are frequently used. The polymerization technique has been often limited by the difficulties in synthesizing and polymerizing highly functionalized monomers while the grafting technique is often limited by the poor stability towards oxygen or water of the reactive polymeric substrates as well as by the need of several synthetic steps generally leading to low chromophore concentrations. In recent years dendrimers have been used to reduce diffusivity of dye molecules in the solid state and to prevent their self-aggregation. In fact, it has been shown that encapsulating individual chromophores can greatly enhance their optical properties. The encapsulation of individual chromophores has been also studied in solutions, by using as host molecules not only dendrimers but also large cyclic compounds, like cyclodextrins, cyclophanes and crown ethers containing many supramolecular optical sensing complexes [1,2].

A more simple alternative method to reduce the diffusivity of chromophore molecules in the solid state and to prevent their self-aggregation consists in the formation of co-crystals with suitable polymer hosts. Particularly efficient and versatile appears to be the encapsulation of chromophore molecules as guest of the host nanoporous crystalline phases (the so called $\delta[3,4]$ and $\varepsilon[5,6]$ phases) of syndiotactic polystyrene (sPS).

The $\delta$ nanoporous crystalline phase presents two identical cavities and eight styrene monomeric units per unit cell [3] (see Figure 1(a)) and is able to sorb volatile organic compounds (both from gas phase and aqueous solutions), also when present at very low concentrations [7]-[9]. The $\delta$ crystalline phase can be considered as the first example of polymeric molecular sieves [10], as it displays a high molecular sorption selectivity which is similar to zeolites. The sorption of suitable guest molecules into the $\delta$ crystalline phase leads to the formation in most cases of clathrate phases, including isolated guest molecules (generally one per cavity, corresponding to a maximum molar ratio guest/host monomer-unit of $1 / 4)[11,12]$, and in few cases of intercalate phases, including layers of guest molecules intercalated with layers of polymer helices (corresponding to maximum molar ratio guest/host monomer-unit of 1/2) [13,14].

The second nanoporous crystalline form of sPS named $\varepsilon$ is characterized by channel-shaped cavities crossing the unit cells along the $c$-axis (see Figure 1(b)) [6], rather than by isolated cavities as observed for the $\delta$ form $[3,4]$. This different 


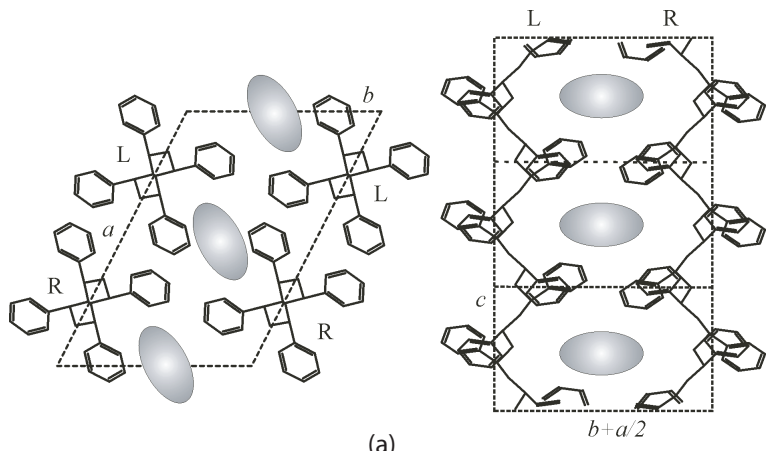

(a)
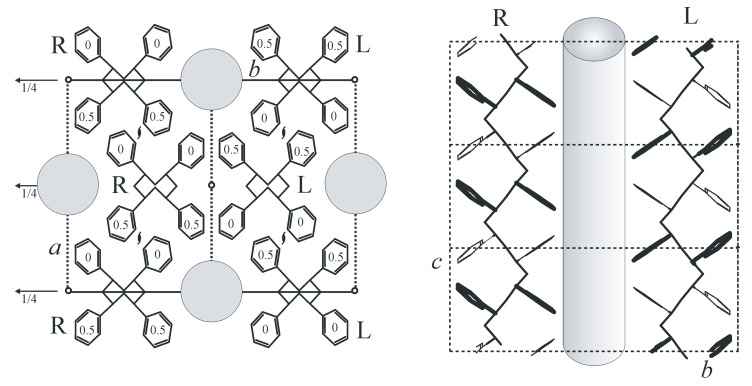

(b)

FIG. 1 Packing models of the nanoporous (a) $\delta$ and (b) $\varepsilon$ crystalline forms of sPS (left: up view, right: lateral view).

type of nanoporosity generally leads, in co-crystals obtained from the $\varepsilon$ phase, to an orientation of the guest molecular planes parallel to the polymer host chain-axes [5] rather than perpendicular, as generally observed $[15,16]$ for co-crystals from the $\delta$ phase. Moreover the channel-shaped cavities of the $\varepsilon$-form allows to obtain co-crystals with guest molecules much longer than those hosted by the $\delta$ "polymeric framework" [5].

Unlike all the traditional procedures that lead to the formation of amorphous phases which display optoelectronic properties and are characterized by a disordered distribution of the photoactive groups, chromophore guest molecules of sPS cocrystals, although with some mobility with respect their minimum energy positions $[17,18]$, present well defined threedimensional order with respect to the crystalline axes [3]-[6]. Moreover, since depending on their preparation procedure sPS co-crystals can assume different kinds of uniplanar orientation [19]-[21], it is possible to control the orientation of the guest molecules not only in the microscopic crystalline phase but also in macroscopic films. This is particularly relevant in the case of active guest molecules (for example nonlinear active, fluorescent, photoreactive, chiral, paramagnetic, etc.) as it is possible to distribute the molecules with a high orientational and positional order.

In the following sections different aspects relative to the structure and the optical properties of sPS films characterized by co-crystalline phases with non-linear optical, fluorescent, photoreactive and chiral guest molecules will be presented and discussed.

\section{CO-CRYSTALS WITH NON-LINEAR OPTICAL GUEST MOLECULES}

In recent studies, it has been shown that the $\delta$ phase of sPS, although apolar, is able to absorb, from solutions in suitable carrier-solvents, high-polarity guests eventually leading to highly stable apolar-host/polar-guest clathrate phases [16]. This procedure allows the preparation of sPS co-crystals with high polarity guests like 4-nitro-aniline $(\mu=6.2 \mathrm{D})$ having non-zero first order hyperpolarizability [16].

In Table 1, are listed benzene derivatives that can form cocrystalline phases with the sPS $\delta$-form.

The size of the $\delta$-form cavities does not allow to form cocrystals with benzene derivatives having a molecular volume larger than the upper limit observed for guests of sPS clathrate phases obtained from $\delta$ phase $\left(V_{m}=0.26 \mathrm{~nm}\right)$ [14] and only benzene derivatives with $\beta$ up to $17 \times 10^{-30}$ esu were found to form co-crystalline phases.

The channel-shaped cavities of the $\varepsilon$-form give the possibility to obtain co-crystalline phases with bulkier guests and molecules with a higher first order hyperpolarizability than trans-4-methoxy- $\beta$-nitrostyrene $\left(\beta=17 \times 10^{-30} \mathrm{esu}\right)$ were found to form co-crystals [5].

In Table 2, are listed some chromophores too bulkier to form a co-crystalline phase with the $\delta$-form but being able to form co-crystals with the sPS $\varepsilon$-form.

The $\varepsilon$-form allows the preparation of sPS co-crystals guest molecules exhibiting relatively high first order hyperpolarizability, like e.g. Disperse Red $1\left(\beta=125 \times 10^{-30} \mathrm{esu}\right)$.

The different shape of the cavity not only allows to obtain cocrystal phases with bulkier molecules but also leads to a different orientation of the guest molecules within the crystalline unit cells.

In Figure 2 are reported for uniaxially stretched sPS films characterized by co-crystalline $\varepsilon /$ p-nitroaniline (pNA) and $\delta /$ pNA phases, the FTIR spectra with polarized light parallel (thin blue lines) and perpendicular (thick red lines) to the film stretching direction.

It is apparent that for both co-crystalline phases, the polymer host peaks present the same dichroism while the sign of the dichroism of pNA peaks is opposite for molecules absorbed in $\delta$-form and $\varepsilon$-form films. This different dichroism is due to a different orientation of the pNA molecules with respect to the chain axis. From the dichroism values it is possible to determine the orientation of guest molecules with respect to the chain axis of the polymeric crystalline host $[15,16]$ and in $\varepsilon /$ pNA co-crystals the dipole moments of the pNA tend to be parallel to the polymer chain axes (see Figure 2(b), left) while in $\delta /$ pNA co-crystals the dipole moments tend to be perpendicular to the chain axes (see Figure 2(b), right).

Through the macroscopic orientation of the polymer host crystalline phase it is possible to obtain a macroscopic orienta- 


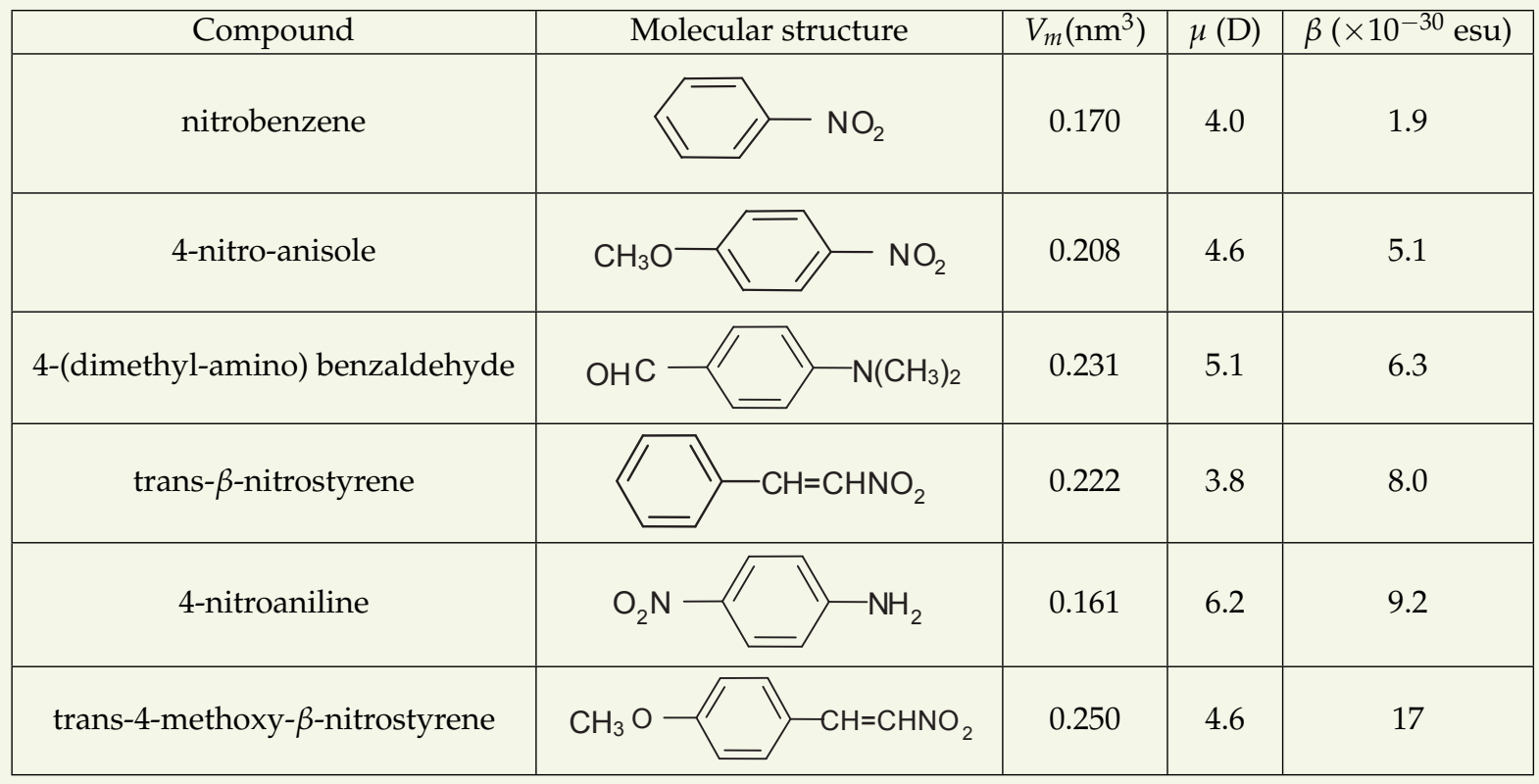

TABLE 1 Molecular structure, molecular volume $\left(V_{m}\right)$, dipole moment $(\mu)$ [22]-[24] and hyperpolarizability $(\beta)$ [22]-[24] values of guest molecules forming co-crystal phases with the $\delta$-form. The molecular volume of the molecules has been calculated from their molecular mass $(M)$ and density $(\rho) V_{m}=M / \rho N_{A}$ where $N_{A}$ is the Avogadro's number $\left(6.02 \times 10^{23} \mathrm{molecules} / \mathrm{mol}\right)$.

\begin{tabular}{|c|c|c|c|c|}
\hline Compound & Molecular structure & $V_{m}\left(\mathrm{~nm}^{3}\right)$ & $\mu(\mathrm{D})$ & $\beta\left(\times 10^{-30} \mathrm{esu}\right)$ \\
\hline $\begin{array}{c}\text { 4-(dimethylammino)- } \\
\text { cinnamaldehyde }\end{array}$ & & & \\
\\
$\begin{array}{c}\text { N,N-Dimethyl-4-nitro-4- } \\
\text { stilbenamine }\end{array}$
\end{tabular}

TABLE 2 Molecular structure, molecular volume $\left(V_{m}\right)$, dipole moment $(\mu)$ [22]-[24] and hyperpolarizability $(\beta)$ [22]-[24] values of molecules forming co-crystal phases only with the $\varepsilon$-form. The molecular volume of the molecules has been calculated from their molecular mass $(M)$ and density $(\rho) V_{m}=M / \rho N_{A}$ where $N_{A}$ is the Avogadro's number $\left(6.02 \times 10^{23} \mathrm{molecules} / \mathrm{mol}\right)$.

tion of the NLO guest molecules. Particularly interesting can be the preferential orientation of the molecular dipoles perpendicular to the film surface, which can be achieved starting from $\varepsilon$-form films presenting the (002) orientation with the chain axes of the host crystalline phase perpendicular to the film plane [5] (see Figure 3). The dipole perpendicular orientation makes these new materials suitable candidates for electrical poling processes, possibly leading not only to non-linear optical but also to ferro-electric and piezoelectric properties.

\section{FLUORESCENT CO-CRYSTALS}

sPS can form co-crystalline phases with various fluorescent chromophores. The formation of these host/guest co-crystals is highly beneficial to the stability of the poly- mer/chromophore systems since it strongly reduces the chromophore diffusivity.

It has been shown that some fluorescent molecules (e.g. naphthalene [25]) can form clathrate co-crystals with sPS while other fluorescent molecules (e.g. 1,4-dimethyl-naphthalene [14]) form intercalate co-crystals. Very recently it has been shown that 1,3,5-trimethyl-benzene (TMB) can form both clathrate and intercalate co-crystals and that these co-crystals show different fluorescence behavior [26].

In Figure 4 are reported the schematic models of the clathrate form (a) and intercalate form (b) of sPS containing TMB.

The fluorescence of a sPS/TMB film ( $9 w t \%$ of TMB) containing the clathrate phase (Figure $4(\mathrm{~d})$, blue thin line) presents a broad maximum nearly centered at $300 \mathrm{~nm}$, which is essen- 


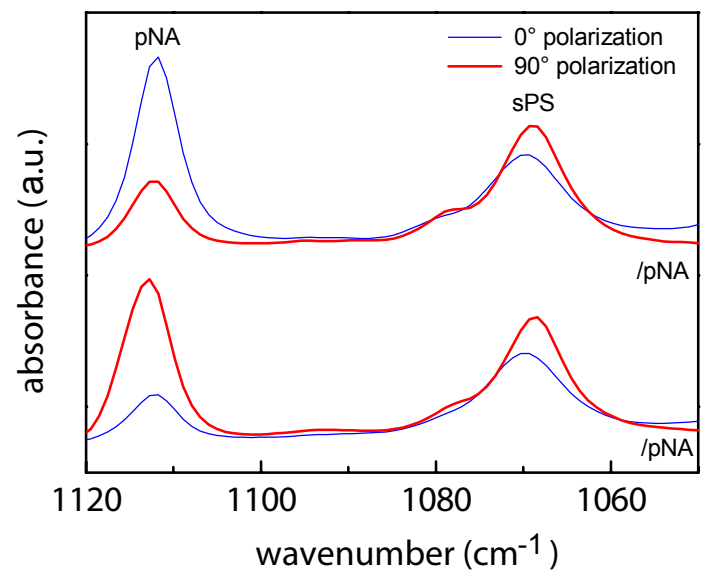

(a)
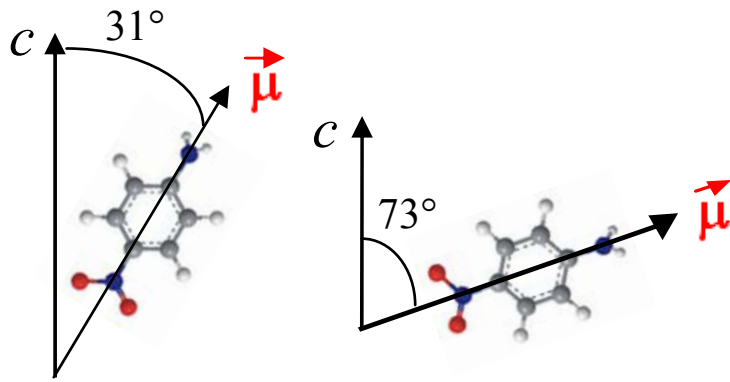

(b)

FIG. 2 (a) FTIR spectra in the wavenumber range $1120-1050 \mathrm{~cm}^{-1}$ taken with the polarization plane parallel (thin blue line) and perpendicular (thick red line) to film stretching direction for $\delta /$ pNA and $\varepsilon /$ pNA co-crystal films. (b) pNA dipole orientation with respect to the $c$-axis of the host polymeric phase in $\varepsilon / p N A$ (left) and in $\delta / p N A$ (right) co-crystals.
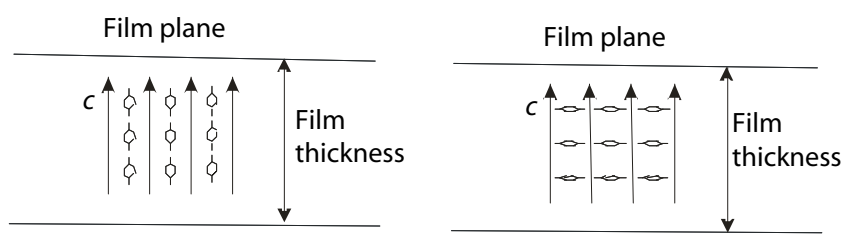

FIG. 3 Schematic presentation of the orientation of the co-crystal chain axes (c) and of NA guest molecules, as obtained by NA absorption in $\varepsilon$-form and $\delta$-form films, presenting an ideal perpendicular (002) orientation.

tially additive with respect to the host (nearly at $315 \mathrm{~nm}$, Figure 4(c)) and the guest (nearly at $290 \mathrm{~nm}$, Figure 4(c)) fluorescence. It is worth adding that the fluorescence is strictly similar to those of films presenting dense polymeric crystalline phases $(\alpha, \beta$ or $\gamma)$ with TMB simply dissolved in their amorphous phases, as well as rather similar to those of fully atactic PS films with TMB.

Unlike the sPS/TMB clathrate phase, the fluorescence of the sPS/TMB film (13 wt $\%$ of TMB) containing the intercalate phase, which is shown as a black thick line in Figure 4(d), presents instead a nearly negligible emission band in the range $270-310 \mathrm{~nm}$ and an intense fluorescence band with three clearly apparent maxima at 330, 340 and $355 \mathrm{~nm}$.

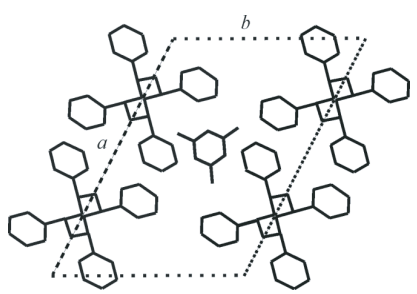

(a)

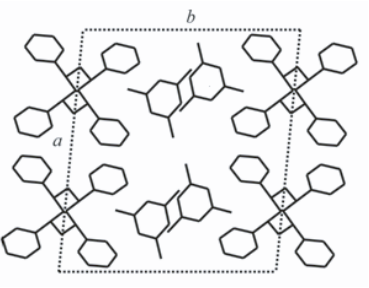

(b)

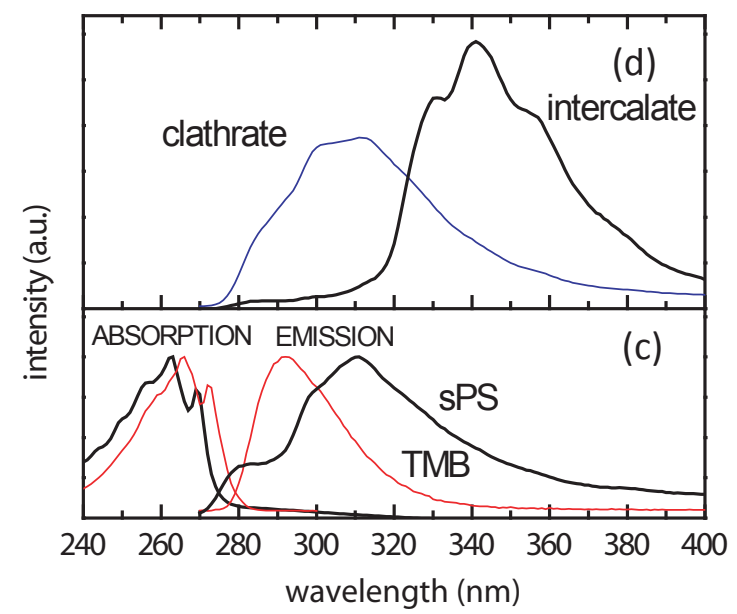

FIG. 4 Schematic models of the projection along the $c$-axis of the clathrate form (a) and intercalate form (b) of sPS containing 1,3,5-trimethyl-benzene. (c) Absorption and emission spectra (excitation at $265 \mathrm{~nm}$ ) of a semicrystalline $\delta$ form sPS film (black thick lines) and of a TMB solution in cyclohexane (red thin lines). (d) Emission spectra (excitation at $265 \mathrm{~nm}$ ) of SPS films presenting clathrate co-crystals (guest content $9 \mathrm{wt} \%$, blue thin line) and intercalate co-crystals: (guest content $13 \mathrm{wt} \%$, black thick line).

This red-shift and fluorescence enhancement could be relevant for optical and optoelectronic applications. Particularly interesting is the ability to emit at longer wavelengths, which could bring the benefit of minimum losses due to reabsorption of the host phase.

\section{PHOTOREACTIVE CO-CRYSTALS: OPTICAL MEMORIES}

Photoisomerizations of organic dyes have been widely used as a means to record optical data. In particular, the photoisomerization of norbornadiene and its derivatives $(\mathrm{N}$, see Figure 5) leading to quadricyclane and its derivatives $(Q$, see Figure 5) has been deeply studied and polymeric materials containing $\mathrm{N}$ derivatives (both as covalently bonded pendant groups or simply added to transparent amorphous phases) have been investigated for optical waveguides (utilizing photo-induced refractive index changes) and for data storage [27]-[29]. However, in all these optical materials there is a complete disorder in the spatial disposition of the photoisomerizing molecules.

In some recent studies it has been reported that norbornadiene could form a clathrate phase with sPS and that efficient 


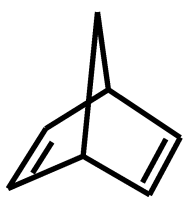

$\mathrm{N}$

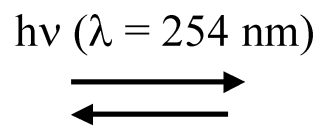

$\Delta$

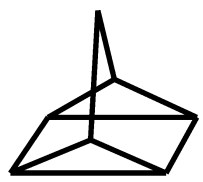

Q
FIG. 5 Photo-isomerization of norbornadiene (N) to quadricyclane (Q).

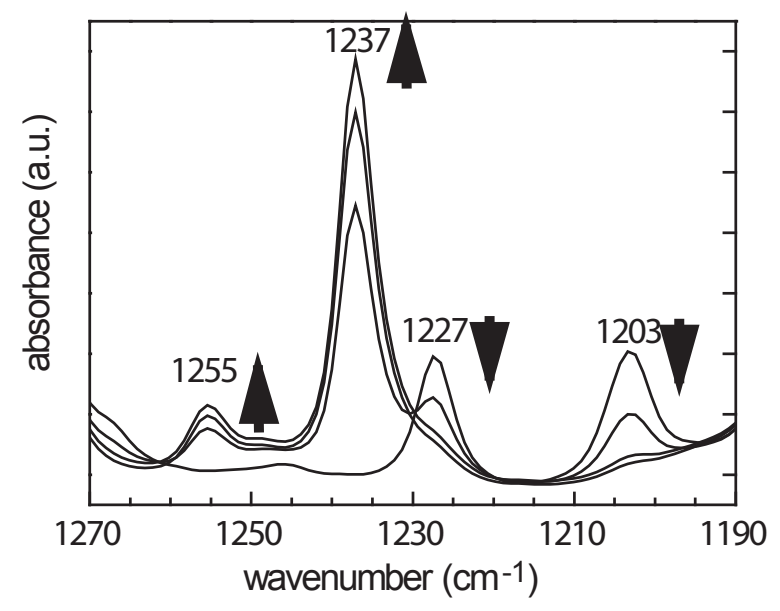

(a)

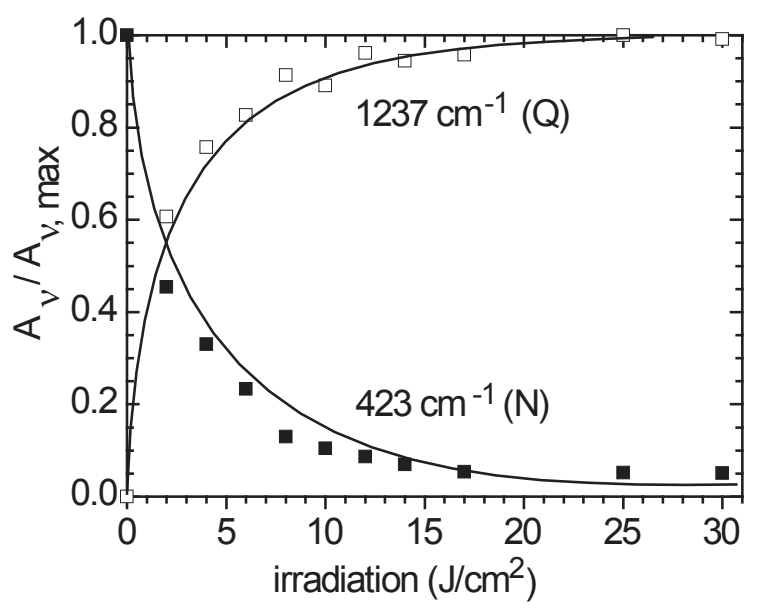

(b)
FIG. 6 (a) FTIR spectra for the range $1270-1190 \mathrm{~cm}^{-1}$, of a SPS film with a clathrate phase containing $\mathrm{N}$, and after subsequent UV (254 nm) irradiation doses. (b) Photoisomerization kinetics of $\mathrm{N}$ to $\mathrm{Q}$, as shown by the absorbance variations of the 423 and $1237 \mathrm{~cm}^{-1}$ peaks.

valence photoisomerization of $\mathrm{N}$ to $\mathrm{Q}$ in the crystalline phase can be achieved $[30,31]$.

In Figure 6(a) are compared the FTIR spectrum for the spectral range $1270-1190 \mathrm{~cm}^{-1}$ of a sPS film characterized by a clathrate phase with $\mathrm{N}$ with the FTIR spectra of the same film, after subsequent irradiation doses at a wavelength of $254 \mathrm{~nm}$.

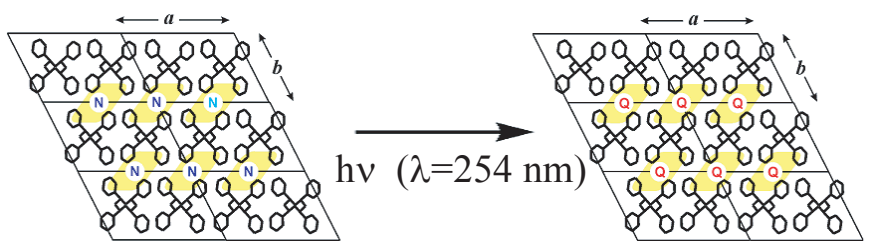

FIG. 7 Positions of the guest molecules in the crystalline clathrate phase, before and after photoisomerization.

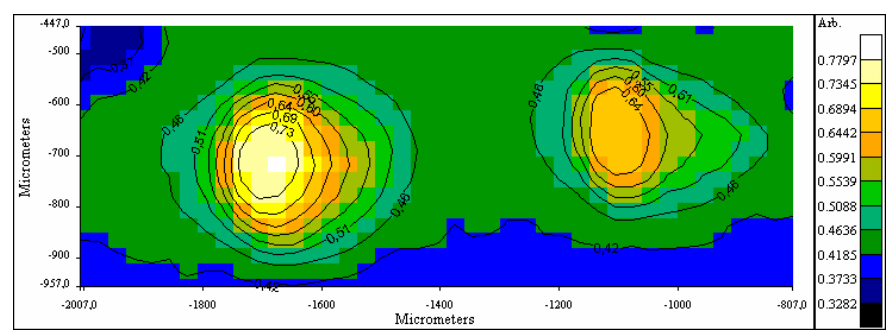

FIG. 8 FTIR of a SPS/N co-crystal film, UV irradiated in the presence of a metal mask relative to the distribution of $\mathrm{Q}$ molecules.

The FTIR spectra clearly show the complete progressive disappearance of the $\mathrm{N}$ absorbance peaks at 1227 and $1203 \mathrm{~cm}^{-1}$ and the appearance of new peaks at 1255 and $1237 \mathrm{~cm}^{-1}$ which can be attributed to quadricyclane [32, 33]. The variation of the $423(\mathrm{~N})$ and $1237 \mathrm{~cm}^{-1}(\mathrm{Q})$ infrared absorbance peaks reported in Figure 6(b) show that complete $\mathrm{N} \rightarrow \mathrm{Q}$ photoisomerization can be realized by UV $(254 \mathrm{~nm})$ irradiation.

It is worth adding that after irradiation and complete $\mathrm{N} \rightarrow \mathrm{Q}$ photoisomerization a co-crystalline phase is still obtained and there is the maintenance of the positions and orientations of the guest molecules as schematically shown in Figure 7 [30].

The FTIR image presenting the distribution of $Q$ molecules after UV irradiation in the presence of a metal mask with a row of holes with a diameter of $200 \mu \mathrm{m}$ and distance between hole centers of $600 \mu \mathrm{m}$ of a sPS/N cocrystal film is reported in Figure 8.

The FTIR image of Figure 8 shows that irradiation experiments, leading to $\mathrm{N} \rightarrow \mathrm{Q}$ photo-isomerization, allow the preparation of micrometric size patterns, by using suitable opaque masks. This suggests that the co-crystalline films are possibly suitable for data storage systems with molecular size marks.

\section{TEMPORARY CO-CRYSTALS WITH NON-RACEMIC GUESTS: OPTICAL SENSORS OF CHIRAL MOLECULES}

Methods for sensing chirality are generally based on nonracemic host receptors (i.e. modified cyclodextrins, cyclophanes, and crown ethers [34, 35] or optically active polymers [36]) interacting with target non-racemic guests. However, these receptors are generally unsuitable for chiral sensing by circular dichroism (CD) measurements, due to their high specificity and their possible intrinsic $C D$. 


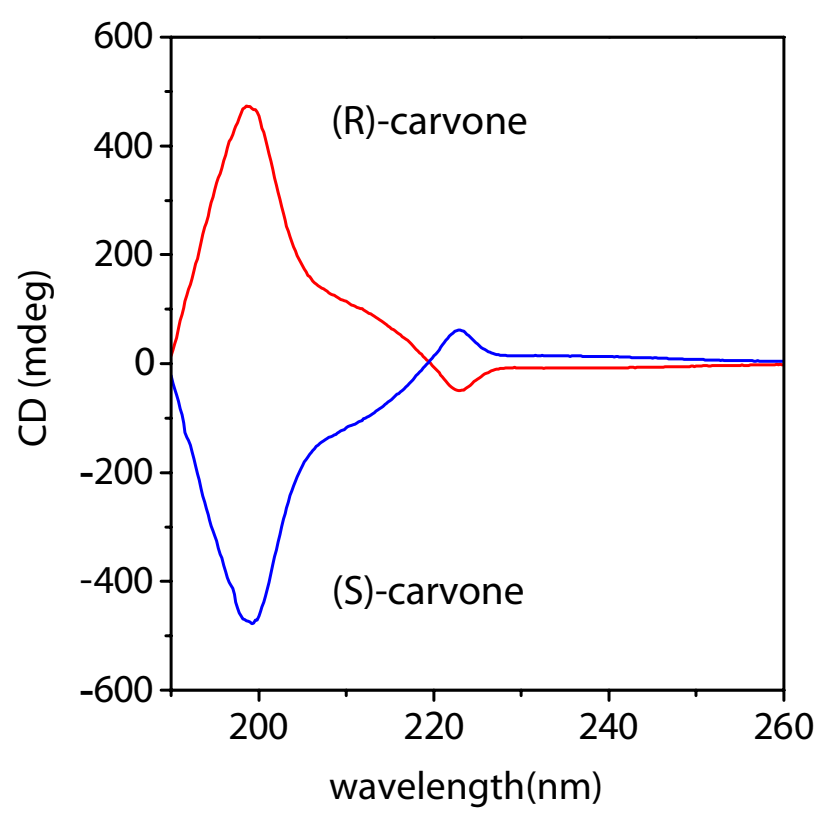

FIG. 9 CD spectra of spin-coated SPS films onto quartz surface, after exposure to vapors of $(\mathrm{R})$ - or (S)-carvone. The spin-coating process has been conducted with $1 \mathrm{wt} \%$ chloroform solutions onto quartz surface at the spin-rate of $1600 \mathrm{rpm}$.

Several methods for sensing chirality based on racemic host receptors interacting with target non-racemic guests have been also proposed. Particularly suitable are macromolecular receptors, being able to form regular helices, and hence are in most cases stereoregular [37]-[42]. In fact, racemic polymers can lead not only to detection but also to amplification of chirality, since cooperative interactions with low-molecular-mass non-racemic compounds can generate prevalence of one polymer helical hand. However these chirality transfer and amplification phenomena have been generally observed in solution [37]-[42].

Recently, it has been observed that syndiotactic polystyrene films prepared by a suitable-spin coating procedure, are able to transfer, amplify and memorize the chirality of non-racemic low-molecular-mass molecules [43, 44].

In Figure 9 are reported the CD spectra of spin-coated sPS films having a thickness of $0.1 \mu \mathrm{m}$ after exposure to the vapor of (R)- and (S)-carvone.

Thus, spin-coated films after exposure to (R)- and (S)-carvone followed by total complete guest removal present a high ICD phenomenon.

A high ICD effect was also observed with several nonracemic molecules which are reported in Figure 10.

The ICD effects like those reported in Figure 9 remain in the polymer film not only after complete guest removal but also after thermal procedures leading to sPS crystal-crystal transitions.

In Figure 11 are reported the CD spectra at room temperature obtained with a film treated with vapors of $\mathrm{R}$ or S-carvone (red lines), and with films treated with vapors of $\mathrm{R}$ or S-carvone

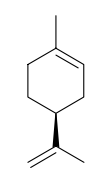

(S)-(-)

Limonene

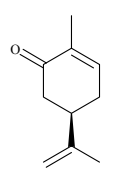

(R)-(-)

Carvone

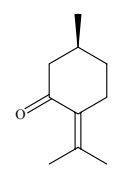

(S)-(-)

Pulegone
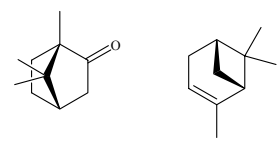

$(1 \mathrm{R})-(-)$

$(1 \mathrm{~S}, 4 \mathrm{~S})-(-)$

$(1 \mathrm{~S}, 4 \mathrm{~S})-(-)$
Camphor

$(1 \mathrm{~S}, 5 \mathrm{~S})-(-)$
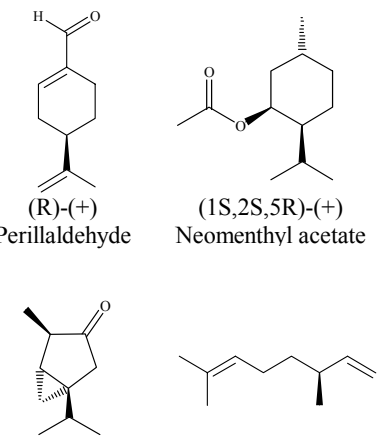

$(1 \mathrm{~S}, 4 \mathrm{R})-(-)$ $\alpha$-Thujone
$(\mathrm{S})-(+)$ $\beta$-Citronellene
FIG. 10 Enantiomers inducing positive ICD effect in spin-coated sPS films.

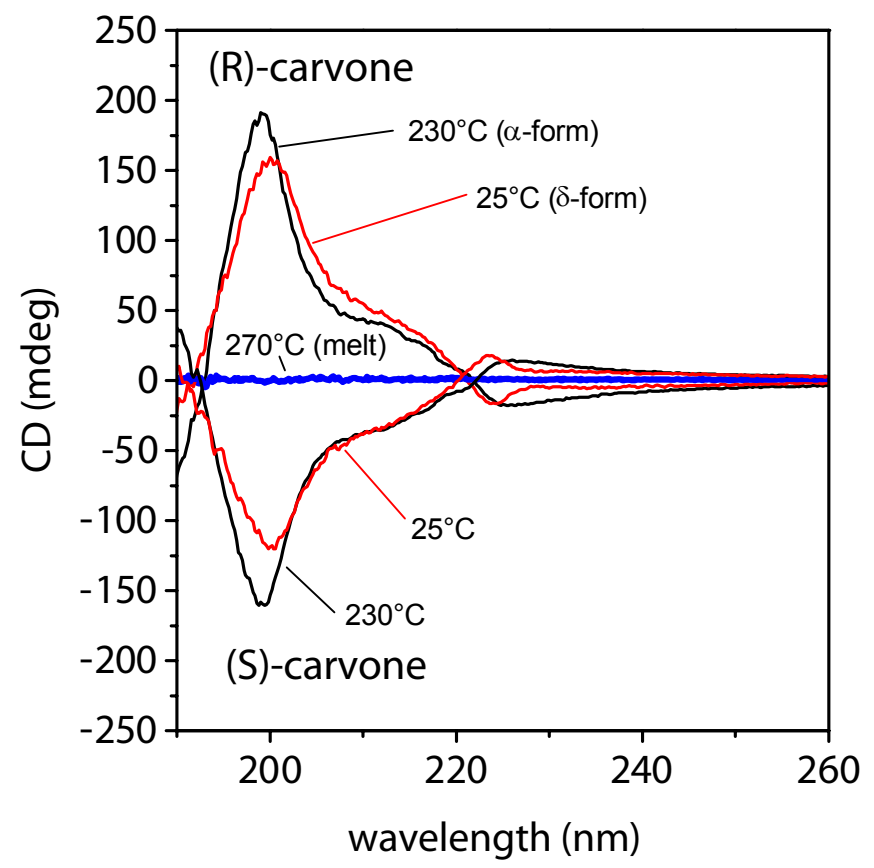

FIG. 11 Room temperature CD spectra of SPS films spin-coated at $1600 \mathrm{rpm}$ from $0.25 \mathrm{wt} \%$ chloroform solution onto quartz surface after: (red lines) exposure to vapors of R or S-carvone; (black lines) exposure to R or S-carvone followed by thermal treatment at $230^{\circ} \mathrm{C}$, leading to crystal-crystal transition from helical toward transplanar crystalline phase; (blue lines) exposure to $\mathrm{R}$ or S-carvone followed by thermal treatment at $270^{\circ} \mathrm{C}$, leading to crystal melting.

and then annealed at $230^{\circ} \mathrm{C}$ (black lines) and at $270^{\circ} \mathrm{C}$ (blue lines).

Figure 11 shows that the ICD effect is maintained at high temperature even after transformation of the $\delta$-form crystalline (characterized by helical chain conformation) into a different crystalline form. In particular after thermal treatments in temperature range $190-230^{\circ} \mathrm{C}$ and formation of the $\alpha$-form crystalline phase (characterized by a trans planar chain conformation) an intense ICD effect is still observed.

The memory of the volatile non-racemic guest molecules can be erased only by thermal treatments at temperatures higher than the sPS melting temperature $\left(\approx 270^{\circ} \mathrm{C}\right)$.

The capacity of syndiotactic polystyrene films, presenting 
a suitable nanoporous host crystalline phase, to transfer, amplify and memorize the chirality of non-racemic lowmolecular-mass molecules associated with a high thermal stability of these ICD phenomena, and hence of the memory of the non-racemic guest molecules suggest a possible application of these polymer films as chiro-optical memories.

\section{CONCLUDING REMARKS}

Polymer films exhibiting co-crystalline phases with low molecular mass chromophores can be suitable for several optical applications. In particular, in this paper, co-crystalline films based on syndiotactic polystyrene (sPS), a commercial semicrystalline polymer with excellent material properties (like, e.g., high melting temperature, high chemical and thermal stability and high crystallization rate), have been presented.

SPS co-crystalline phases can be obtained with non-linear optical, fluorescent, photoreactive and chiral guest molecules. Host-guest co-crystals reduce guest diffusivity and prevent guest self-aggregation, without recurring to chemical reactions. Moreover, by controlling the orientation of the host polymeric crystalline phase it is possible to control the macroscopic three-dimensional orientation of the chromophore guests in the polymer films. These properties make sPS films presenting co-crystals with chromophores potentially interesting as advanced fluorescent, photo-reactive, non-linear optical and chiro-optical materials.

\section{ACKNOWLEDGEMENTS}

Financial support of the "Ministero dell'Istruzione, dell'Università e della Ricerca" (PRIN2007), of Regione Campania (Centro di Competenza), and of INSTM (PRISMA $01 / 2007$ ) is gratefully acknowledged.

\section{References}

[1] A. Douhal, "Ultrafast Guest Dynamics in Cyclodextrin Nanocavities" Chem. Rev. 104, 1955-1976 (2004).

[2] C. Lin, Y. Liu, C. Lai, S. Peng, and S. Chiu, "An Extremely Stable Host-Guest Complex that Functions as a Fluorescence Probe for Calcium Ions" Chemistry 2, 4594-4599 (2006).

[3] C. De Rosa, G. Guerra, V. Petraccone, and B. Pirozzi, "Crystal Structure of the Emptied Clathrate Form ( $\delta_{e}$ Form) of Syndiotactic Polystyrene" Macromolecules 30, 4147-4152 (1997).

[4] G. Milano, V. Venditto, G. Guerra, L. Cavallo, P. Ciambelli, and D. Sannino, "Shape and Volume of Cavities in Thermoplastic Molecular Sieves Based on Syndiotactic Polystyrene" Chem. Mater. 13, 1506-1511 (2001).

[5] P. Rizzo, C. Daniel, A. De Girolamo Del Mauro, and G. Guerra, “New Host Polymeric Framework and Related Polar Cuest Cocrystals" Chem. Mater. 19, 3864-3866 (2007).

[6] V. Petraccone, 0. Ruiz de Ballesteros, 0. Tarallo, P. Rizzo, and G. Guerra, "Nanoporous Polymer Crystals with Cavities and Channels" Chem. Mater. 20, 3663-3668 (2008).
[7] C. Manfredi, M. A. Del Nobile, G. Mensitieri, G. Guerra, and M. Rapacciuolo, "Vapor Sorpion in Emptied Clathrate Samples of Syndiotactic Polystyrene" J. Polym. Sci 35, 135-140 (1997).

[8] C. Daniel, D. Alfano, V. Venditto, S. Cardea, E. Reverchon, D. Larobina, G.Mensitieri, and G. Guerra, "Aerogels with a Microporous Crystalline Host Phase" Adv. Mater. 17, 1515-1518 (2005).

[9] C. Daniel, D. Sannino, and G. Guerra, "Syndiotactic Polystyrene Aerogels: Adsorption in Amorphous Pores and Absorption in Crystalline Nanocavities" Chem. Mater. 20, 577-582 (2008).

[10] G. Guerra, G. Milano, V. Venditto, P. Musto, C. De Rosa, and L. Cavallo, "Thermoplastic Molecular Sieves" Chem. Mater. 12, 363368 (2000).

[11] Y. Chatani, T. Inagaki, Y. Shimane, T. Ijitsu, T. Yukimori, and H. Shikuma, "Structural Study on Syndiotactic Polystyrene: 2. Crystal Structure of Molecular Compound with Toluene" Polymer 34, 16201624 (1993).

[12] C. De Rosa, P. Rizzo, 0. Ruiz de Ballesteros, V. Petraccone, and G. Guerra, "Crystal Structure of the Clathrate $\delta$ Form of Syndiotactic Polystyrene Containing 1,2-Dichloroethane" Polymer 40, 2103-2110 (1999).

[13] V. Petraccone, 0. Tarallo, V. Venditto, and G. Guerra, "An Intercalate Molecular Complex of Syndiotactic Polystyrene" Macromolecules 38, 6965-6971 (2005).

[14] 0. Tarallo, V. Petraccone, V. Venditto, and G. Guerra, "Crystalline Structures of Intercalte Molecular Complexes of Syndiotactic Polystyrene with Two Fluorescent Guests: 1,3,5-Trimethyl-benzene and 1,4-dimethyl-naphthalene" Polymer 47, 2402-2410 (2006).

[15] A. R. Albunia, G. Milano, V. Venditto, and G. Guerra, "A Clear-Cut Experimental Method to Discriminate between In-Plane and Outof-Plane Molecular Transition Moments" J. Am. Chem. Soc. 127, 13114-13115 (2005).

[16] C. Daniel, N. Galdi, T. Montefusco, and G. Guerra, "Syndiotactic Polystyrene Clathrates with Polar Guest Molecules" Chem. Mater. 19, 3302-3308 (2007).

[17] E. Trezza, and A. Grassi, "Dynamics of aromatic molecules clathrated in crystalline Syndiotactic Polystyrene: A Solid State ${ }^{2} \mathrm{H}$ NMR Investigation of the Host/Guest Complexes" Macromol. Rapid Commun. 23, 260-263 (2002).

[18] A. R. Albunia, R. Graf, G. Guerra, and H. W. Spiess, “2H NMR Study of Aromatic Guest Dynamics in Clathrate Phases of Syndiotactic Polystyrene" Macromol. Chem. Phys. 206, 715-7-24 (2005).

[19] P. Rizzo, M. Lamberti, A. R. Albunia, 0. Ruiz de Ballesteros, and G. Guerra, "Crystalline Orientation in Syndiotactic Polystyrene Cast Films" Macromolecules 35, 5854-5860 (2002)

[20] P. Rizzo, A. Costabile, and G. Guerra, "Perpendicular Orientation of Host Polymer Chains in Clathrate Thick Films" Macromolecules 37, 3071-3076 (2004).

[21] P. Rizzo, A. Spatola, A. De Girolamo Del Mauro, and G. Guerra, "Polymeric Films with Three Different Uniplanar Crystalline Phase Orientations" Macromolecules 38, 10089-10094 (2005).

[22] L. -T. Cheng, W. Tam, S. H. Stevenson, G. R. Meredith, G. Rikken, and S. R. Marder, "Experimental Investigations of Organic Molecular Nonlinear Optical Polarizabilities. 1. Methods and Results on Benzene and Stilbene Derivatives" J. Phys. Chem. 95, 10631-10643 (1991).

[23] L. -T. Cheng, W. Tam, S. R. Marder, A. E. Stiegman, G. Rikken, and C. W. Spangler, "Experimental Investigations of Organic Molecular Nonlinear Optical Polarizabilities. 2. A Study of Conjugation Dependences" J. Phys. Chem. 95, 10643-10652 (1991). 
[24] H. E. Katz, K. D. Singer, J. E. Sohn, C. W. Dirk, L. A. King, and H. M. Gordon, "Greatly Enhanced Second-order Nonlinear Optical Susceptibilities in Donor-acceptor Organic Molecules" J. Am. Chem. Soc. 109, 6561-6563 (1987).

[25] V. Venditto, G. Milano, A. De Girolamo Del Mauro, G. Guerra, J. Mochizuki, and H. Itagaki, "Orientation and Microenvironment of Naphthalene Guest in the Host Nanoporous Phase of Syndiotactic Polystyrene" Macromolecules 38, 3696-3702 (2005)

[26] A. De Girolamo Del Mauro, M. Carotenuto, V. Venditto, V. Petraccone, M. Scoponi, and G. Guerra, "Fluorescence of Syndiotactic Polystyrene/Trimethylbenzene Clathrate and Intercalate CoCrystals" Chem. Mater. 19, 6041-6046 (2007).

[27] S. Takahashi, K. Samata, H. Muta, S. Machida, and K. Horie, "Refractive-index Patterning Using Near-field Scanning Optical Microscopy" Appl. Phys. Lett. 78, 13-15 (2001).

[28] T. Nagai, M. Shimada, Y. Ono, and T. Nishikubo, "Synthesis of New Photoresponsive Polymers Containing Trifluoromethyl-Substituted Norbornadiene Moieties" Macromolecules 36, 1786-1792 (2003).

[29] D. R. Robello, S. Y. Farid, J. P. Dinnocenzo, and J. G. Gillmore, “Optical Recording Material”, US patent 6969578 (2005).

[30] P. Stegmaier, A. De Girolamo Del Mauro, V. Venditto, and G. Guerra, "Optical Recording Materials Based on Photoisomerization of Guests Molecules of a Polymeric Crystalline Host Phase" Adv. Mater. 17, 1166-1168 (2005).

[31] C. D'Aniello, P. Musto, V. Venditto, and G. Guerra, "Photoisomerization Patterns Based on Molecular Complex Phases of Syndiotactic Polystyrene" J. Mater. Chem. 17, 531-537 (2007).

[32] D. G. Lishan, K. V. Reddy, G. S. Hamond, and J. E. Leonard, "Overtone Vibrational Photochemistry of Quadricyclane" J. Phys. Chem. 92, 656-660 (1988).

[33] N. T. Kawai, F. R. Gilson, and I. S. Butler, "Variable-Temperature and -Pressure Studies of the Vibrational Spectra and Phase Transition in Quadricyclane" J. Phys. Chem. 96, 8556-8561 (1992).

[34] L. J. Prins, D. N. Reinhoudt, and P. Timmerman, “Noncovalent Syn- thesis Using Hydrogen Bonding" Angew. Chem. Int. Ed. 40, 23822426 (2001).

[35] X. X. Zhang, J. S. Bradshaw, and R. M. Izatt, "Enantiomeric Recognition of Amine Compounds by Chiral Macrocyclic Receptors" Chem. Rev. 97, 3313-3361 (2007).

[36] Y. Okamoto, E. Yashima, and C. Yamamoto, "Optically Active Polymers with Chiral Recognition Ability" Top. Stereochem. 24, 157-208 (2003).

[37] M. M. Green, C. Khatri, and N. C. Peterson, "A Macromolecular Conformational Change Driven by a Minute Chiral Solvation Energy" J. Am. Chem. Soc. 115, 4941-4942 (1993).

[38] A. E. Rowan, R. J. M. Nolte, "Helical Molecular Programming" Angew. Chem. Int. Ed 110, 6368 (1998)

[39] E. Yashima, K. Maeda, and Y. Okamoto, "Memory of Macromolecular Helicity Assisted by Interaction with Achiral Small Molecules" Nature 399, 449-451 (1999)

[40] M. M. Green, J. W. Park, T. Sato, A. Teramoto, S. Lifson, R. L. B. Selinger, and J. V. Selinger, "The Macromolecular Route to Chiral Amplification" Angew. Chem. Int. Ed. 38, 3138-3154 (1999).

[41] H. Nakashima, J. R. Koe, K. Torimitsu, and M. Fujiki, "Transfer and Amplification of Chiral Molecular Information to Polysilylene Aggregates" J. Am. Chem. Soc. 123, 4847-4848 (2001).

[42] E. Yashima, K. Maeda, and T. Nishimura, "Detection and Amplification of Chirality by Helical Polymers" Chem. Eur. J. 10, 42-51 (2004).

[43] A. Buono, P. Rizzo, I. Immediata, and G. Guerra, "Detection and Memory of Nonracemic Molecules by a Racemic Host Polymer Films" J. Am. Chem. Soc. 129, 10992-10993 (2007).

[44] L. Guadagno, M. Raimondo, C. Silvestre, I. Immediata, P. Rizzo, and G. Guerra, "Processing, Thermal Stability and Morphology of Chiral Sensing Syndiotactic Polystyrene Films" J. Mater. Chem. 18, 567-572 (2008). 